\title{
Tentorial Tear Related to Birth
}

National Cancer Institute

\section{Source}

National Cancer Institute. Tentorial Tear Related to Birth. NCI Thesaurus. Code C116831.

Disruption of the tentorium cerebelli incurred during delivery. 